\title{
Precision Capsular Infarct Modeling to Produce Hand Motor Deficits in Cynomolgus Macaques
}

\author{
Hyung-Sun Kim ${ }^{1}$, Jeong Ho Hwang ${ }^{1}$, Su-Cheol Han ${ }^{1}$, Goo-Hwa Kang', \\ Ji-Young Park ${ }^{2}$ and Hyoung-Ihl Kim ${ }^{2,3 *}$ \\ ${ }^{1}$ Animal Model Research Group, Jeonbuk Branch Institute, Korea Institute of Toxicology, Jeongup 53212, \\ ${ }^{2}$ Neuromodulation Lab, Department of Biomedical Science and Engineering, Gwangju Institute of Science and Technology, \\ Gwangju 61005, ${ }^{3}$ Department of Neurosurgery, Presbyterian Medical Center, Jeonju 54987, Korea
}

Stroke research in non-human primates (NHPs) with gyrencephalic brains is a critical step in overcoming the translational barrier that limits the development of new pharmaceutical and rehabilitative strategies for stroke. White-matter stroke (WMS) has a unique pathophysiology from graymatter stroke and is not well understood because of a lack of pertinent animal models. To create a precise capsular infarct model in the cynomolgus macaque, we first used electrical stimulation to map hand movements, followed by viral tracing of the hand motor fibers (hMFs). This enabled us to identify stereotactic targets in the posterior limb of the internal capsule (PLIC). Neural tracing showed that hMFs occupy the full width of the PLIC, owing to overlap with the motor fibers for the leg. Furthermore, the hMFs were distributed in an oblique shape, requiring coronal tilting of the target probe. We used the photothrombotic infarct lesioning technique to precisely destroy the hMFs within the internal capsule. Double-point infarct lesioning that fully compromised the hMFs resulted in persistent hand motor and walking deficits whereas single-point lesioning did not. Minor deviations in targeting failed to produce persistent motor deficits. Accurate stereotactic targeting with thorough involvement of motor fibers is critical for the production of a capsular infarct model with persistent motor deficits. In conclusion, the precision capsular infarct model can be translated to the NHP system to show persistent motor deficits and may be useful to investigate the mechanism of post-stroke recovery as well as to develop new therapeutic strategies for the WMS.

Key words: Stroke, Internal capsule, Cynomolgus macaque, White matter

\section{INTRODUCTION}

White-matter infarcts caused by the obstruction of deep penetrating vessels and end-arteries in the brain represent $20 \sim 30 \%$ of all stroke infarcts [1]. Recent advances in imaging techniques, such as high-resolution MRI, provide an increasing chance of detecting white-matter infarcts associated with cognitive and physical impairments in elderly people [1-3]. In particular, white-matter

Submitted August 10, 2021, Revised September 2, 2021,

Accepted September 30, 2021

* To whom correspondence should be addressed.

TEL: 82-62-715-3234, FAX: 82-62-715-5309

e-mail:hyoungihl@gist.ac.kr infarcts affecting the corticofugal fibers often produce motor-sensory deficits with poor clinical recovery, despite the small extent of the infarct [4]. Until recently, stroke research has been centered exclusively on the pathogenesis and recovery mechanisms of large-artery gray-matter strokes [5]. Many therapeutic regimes and drugs have been developed using gray-matter stroke models [6]. However, neuroscientific research into white-matter strokes is relatively rare in small animals and non-human primates (NHPs, e.g., macaques) owing to the lack of pertinent animal models [7].

Recently, we reported a capsular infarct model created by circumscribed photothrombotic destruction of the internal capsule in rodents after somatotopic mapping of the forelimb motor area $[8,9]$. This model shows long-term, persistent motor deficits, and has been used to investigate the mechanisms of post-stroke recov- 
Table 1. Summary of experimental animals

\begin{tabular}{|c|c|c|c|c|c|c|c|c|c|}
\hline \multirow[b]{2}{*}{ Subject } & \multirow[b]{2}{*}{ Group } & \multirow[b]{2}{*}{ Sex } & \multicolumn{2}{|c|}{ Body weight (kg) } & \multirow[b]{2}{*}{$\begin{array}{c}\text { Post- } \\
\text { operative } \\
\text { mortality }\end{array}$} & \multirow[b]{2}{*}{$\begin{array}{c}\text { Infarct } \\
\text { volume } \\
\left(\mathrm{mm}^{3}\right)\end{array}$} & \multicolumn{3}{|c|}{ Post-infarct evaluation } \\
\hline & & & Pre-infarct & Necropsy & & & $\begin{array}{l}\text { Neurological } \\
\text { score }\end{array}$ & $\begin{array}{c}\text { Brinkman } \\
\text { board } \\
\text { score }(\%)\end{array}$ & $\begin{array}{c}\text { Walking } \\
\text { deficit }\end{array}$ \\
\hline 1 & Neural tracing & Male & 5.528 & 5.784 & No & & & & \\
\hline 2 & SPL & Male & 5.378 & 5.715 & No & 4.747 & 0 & 100 & _- \\
\hline 3 & DPL-DL & Male & 3.879 & 4.092 & No & 3.151 & 1 & 30 & - \\
\hline 4 & DPL-DL & Male & 3.554 & 4.039 & No & 9.303 & 1 & 51.7 & - \\
\hline 5 & DPL-CL & Male & 5.074 & 5.016 & No & 21.075 & 8.5 & 0 & + \\
\hline 6 & DPL-CL & Male & 5.249 & 5.634 & No & 14.809 & 6.8 & 0 & + \\
\hline 7 & DPL-CL & Male & 3.805 & 4.041 & No & 22.242 & 3 & 0 & + \\
\hline 8 & DPL-CL & Male & 3.115 & 3.413 & No & 23.717 & 6 & 0 & + \\
\hline
\end{tabular}

ery and changes in brain activity and behavior with the application of a variety of neuromodulatory technologies [10-14]. However, a translational barrier exists between rodents and humans because of the anatomical and physiological differences between the two species. In rodents, the axons of corticospinal neurons heavily innervate subcortical targets, such as the striatum, thalamus, subthalamic nucleus, ventral midbrain and brain stem whereas corticospinal neurons sparsely branched to the striatum and claustrum without innervating to other forebrain or midbrain regions in primates [15]. These differences often leads to the frequent failure of preclinical studies [16]. Therefore, a capsular stroke model in a gyrencephalic NHP would have great value [16].

In this study, we describe a subcortical capsular infarct model in cynomolgus macaque produced by targeted photothrombotic lesioning. To determine the extent of the infarct lesioning, we first performed motor mapping of the hand area in the primary motor cortex, followed by injection of adeno-associated virus (AAV5CamKII-EYFP) to identify the location of the corticospinal tract in the posterior limb of the internal capsule (PLIC). A cannula was stereotactically introduced parallel to the oblique trajectory of the internal capsule to compromise the maximum number of hand motor fibers in the PLIC during unilateral photothrombotic infarct lesioning. This model produced long-term motor deficits that can be used to investigate white-matter stroke in macaques, particularly post-stroke recovery and translational research. We also discuss the confounding factors relevant to this precise capsular infarct model.

\section{MATERIALS AND METHODS}

\section{Animals}

All animals were managed in accordance with the guidelines of the Association for the Assessment and Accreditation of Laboratory Animal Care (AAALAC). Experimental animals were housed one per cage in a controlled animal facility at $21 \pm 1^{\circ} \mathrm{C}$ with water ad libitum. The animal care unit was maintained on a 12-hour light-dark cycle with lights on at 8:00 am.

Animal experiments were conducted after approval by the Institutional Animal Care and Use Committee (IACUC) of the Korean Institute of Toxicology (KIT-1509-0275, KIT-1607-0262, KIT1705-0176, KIT-1801-0034, KIT-2007-0222) and GIST (Gwangju Institute of Science and Technology). Eight macaques (male, 3.1 to $5.5 \mathrm{~kg}, 3$ to 5 years in age) were used for this study: one for neural tracing and seven for photothrombotic capsular infarct modeling in the PLIC. Single point infarct lesioning (SPL) was conducted in one macaque and double-point infarct lesioning (DPL) was conducted in six macaques (Table 1). After histological examination, the six DPL macaques were subdivided into a "correct lesioning" group (DPL-CL; $\mathrm{n}=4$ ), with accurate targeting in the PLIC, and a "deviated lesioning" group (DPL-DL; $\mathrm{n}=2$ ).

\section{Neural tracing from hand motor fibers in the internal capsule}

To verify the exact location of the corticospinal tract associated with hand movements within internal capsule, we performed electrical motor mapping in the motor cortex. Under general anesthesia with $2 \%$ isoflurane, the macaquess head was fixed in a stereotactic frame while vital signs were monitored and body temperature was maintained at $\sim 37^{\circ} \mathrm{C}$. The scalp was incised and a craniotomy was performed over the central area of the lateral hemisphere. After exposing the dura, the hand motor area was mapped by applying a unipolar current $(5 \mathrm{~Hz}, 5 \mathrm{~V}, 5 \mathrm{sec})$ to the cortex while observing contralateral hand movements (supplementary 1). If stimulation of an area induced hand movement, that cortical area was designated as part of the hand area of the motor cortex (Fig. 1A).

Neural tracing was conducted with adeno-associated virus (AAV5-CaMKII-EYFP, virus core facility, KIST, Seoul, Korea). 30 
A.

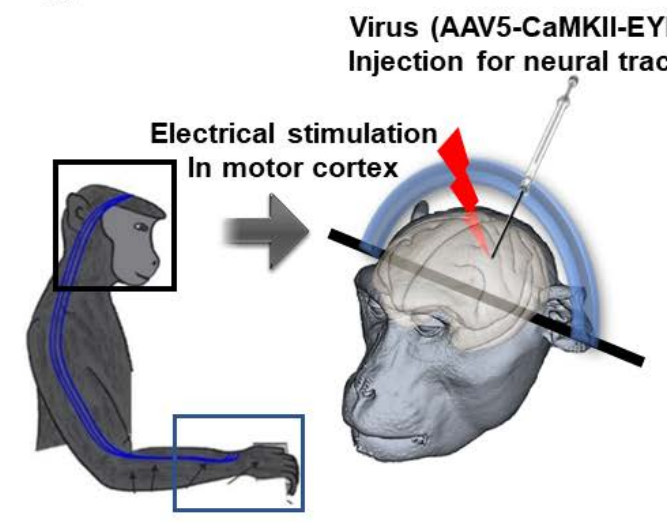

Finger \& hand movement
B.

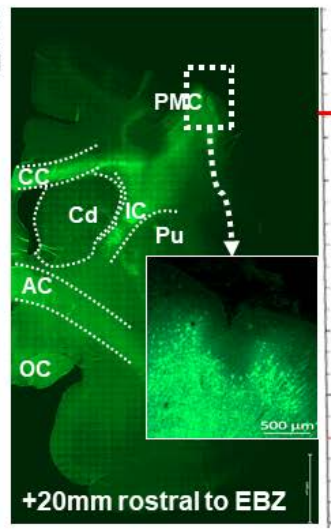

C.



D.



Fig. 1. Identification of hand motor fibers. (A) Schematic drawing showing the electrical stimulation of motor cortex and simultaneous observation of stimulation-induced hand movements. The viral construct (AAV5-CaMKII-EYFP) was injected at the site of stimulation. (B D) The coronal sections showing the viral expression of injection site and hand motor fibers were shown, and transcribed into the stereotactic atlas. AC, anterior commissure; Cd, caudate; CC, corpus callosum; GPe, globus pallidus externus; GPi, globus pallidus internus; IC, internal capsule; PMC, primary motor cortex; Pu, putamen; Thal, thalamus.

$\mu \mathrm{l}$ of AAV was injected into hand area of the motor cortex at a rate of $2.5 \mu \mathrm{l} / \mathrm{min}$ with a $26 \mathrm{G}$ Hamilton syringe connected to an UltraMicroPump (WPI, Sarasota, FL, USA). After infusion, the microcannula was left at the injection site for an additional 10 minutes before being slowly removed. After closure of operative wounds, the macaque was released from the stereotactic apparatus, given antibiotic and analgesic injections [cephazolin (20 mg/kg), ketoprofen $(3 \mathrm{mg} / \mathrm{kg})]$, and transferred to the recovery cage. After waiting 6 weeks for viral expression, the macaque was sacrificed and $4 \%$ paraformaldehyde solution (PFA) was perfused transcardially for $20 \mathrm{~min}$. Then, the brain was removed and further fixed by immersion in $4 \%$ PFA for $24 \mathrm{~h}$. Brains were sectioned coronally at 40 $\mu \mathrm{m}$ thickness and mounted onto glass slides. Images were acquired with a DM3000 microscope (Leica, Germany), a Zeiss LSM 800 confocal microscope, and Zeiss Axioscan Z1 slide scanner (Zeiss, Germany). The EYFP expression was transcribed into the stereotactic atlas to show the relative location of the hand motor fibers in the PLIC (Fig. 1B 1D). These drawings were independently confirmed by three reviewers.

\section{Internal capsule infarction using photothrombosis}

On the basis of the neural tracing results, we determined the coordinates of the stereotactic target for photothrombotic infarct lesioning as the region of the PLIC in which hand motor fibers were distributed. The unilateral photothrombotic infarct lesioning procedure was similar to that previously described [9]. Briefly, the monkey's head was fixed in the "stereotactic frame with simplified adaptor" [17] in which coronal tilt is feasible. A scalp incision and skull opening were made to allow the optical fiber cannula (OFC; a $400 \mu \mathrm{m}$ optical fiber placed inside an 18 gauge spinal needle) to be lowered to the target position; the other end of the OFC was connected to a green laser system $(532 \mathrm{~nm}$; Shanghai Laser and Optics Century, Shanghai, China) (Fig. 2A).

After a durotomy, the OFC was inserted stereotactically into the PLIC for single (SPL) and double (DPL) targets. The SPL target was the center of the PLIC (10 mm lateral to the midline, $14 \mathrm{~mm}$ rostral to the interaural-EBZ line, $14 \mathrm{~mm}$ dorsal to the ear bar, inclination angle of $32^{\circ}$ ) (Fig. 2B). For DPL, the deeper location was targeted first, with the second target $3 \mathrm{~mm}$ above the first (Fig. 2C). Rose bengal (20 mg/kg, Sigma-Aldrich, Saint-Louis, U.S.A) was administered intravenously while body temperature was maintained at $37^{\circ} \mathrm{C}$. For SPL, the target site was irradiated for 5 min with a green laser $(532 \mathrm{~nm}, 100 \mathrm{~mW}$ intensity at the fiber tip). For DPL, two PLIC irradiations were performed, keeping the inclination angle at $32^{\circ}$. After all procedures were complete, the OFC was withdrawn and the animals were provided postoperative care as described above. All the macaques tolerated the operative procedures with no perioperative deaths and did no decline in body weight (Table 1).

\section{Behavioral evaluation}

Two types of behavioral tests were used in this study: a neurological assessment with a stroke rating scale for quantification of neurological deficits [18], and the Brinkman board measure of manual dexterity [19]. For the neurological assessment, the scale was weighted heavily toward motor functions, including grasp, 
A.

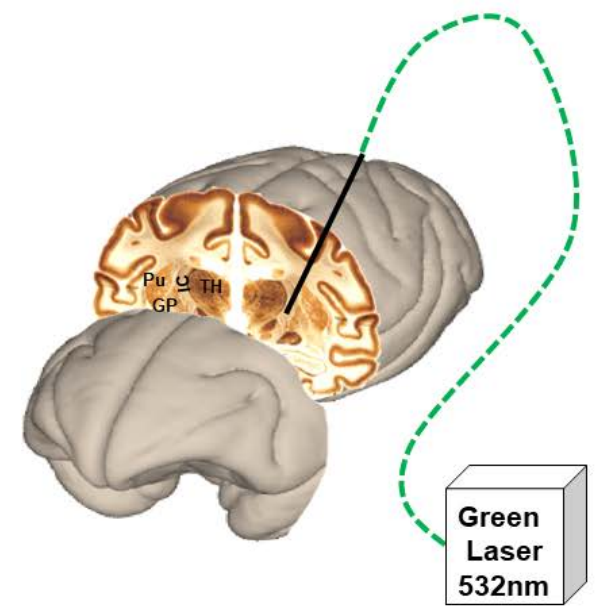

Capsular infarct lesioning
B.

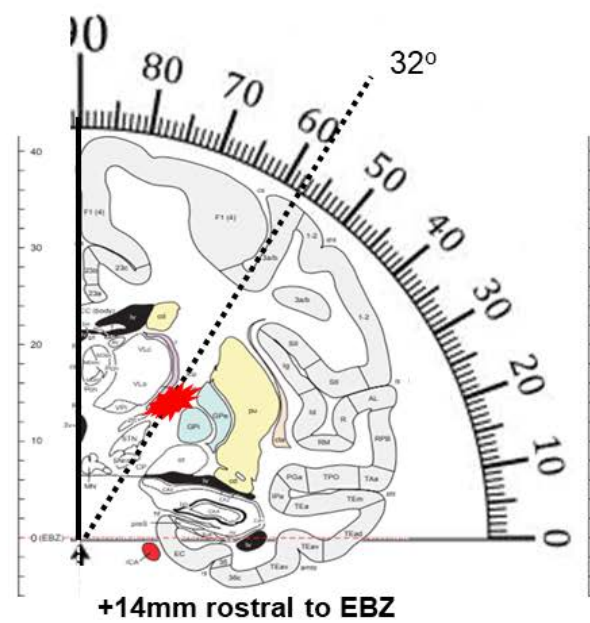

Single point lesion (SPL) c.

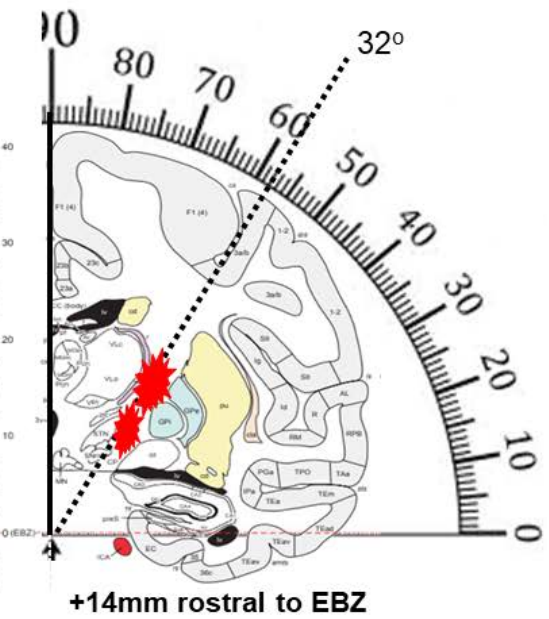

Double point lesion (DPL)

Fig. 2. Stereotactic targeting for induction of photothrombotic infarct lesioning. (A) Schematic drawing showing the introduction of the optical fiber cannula into the posterior limb of the internal capsule. (B, C) Schematic drawings showing stereotactic targeting of the hand motor fibers at a single site (B) and at double sites $(\mathrm{C})$.

movement of extremities, gait, circling, balance, bradykinesia, and facial weakness, as well as assessing consciousness, visual field, and neglect. From a total of 41 points, 0 corresponds to normal behavior and 41 corresponds to severe bilateral neurological impairment. Neurological assessment scores were evaluated three times per week by the same trained technician.

For the Brinkman board test, macaques were placed in a cage in which different window provide access for the right and left hands. The Brinkman board consisted of 20 slots containing chocolatecoated sunflower seeds and was presented to the macaque's contralesional hand for free retrieval (supplementary 2). After the test, number of pellets remaining in the slots was counted for analysis. The Brinkman board test was repeated three times per week before and after infarct lesioning until behavioral evaluation was terminated. Videos of all tests were recorded for later analysis.

\section{Histology and MRI imaging}

Experimental animals were euthanized with an overdose of thiopental sodium six weeks after neural tracing and three months after capsular infarct lesioning. Transthoracic cardiac perfusion was conducted as described above. Nissel staining and immunohistochemical staining for glial fibrillary acidic protein (GFAP) were performed as described in previous reports $[8,9]$. The infarction volume was measured in each animal with Image (NIH, Bethesda, MD, USA) [8].

In addition, two macaques (one SPL and one DPL-CL) underwent magnetic resonance imaging (MRI, Magnetom Verio 3T,
Siemens Healthcare, Munich, Germany) six months after capsular lesioning to verify the long-term changes in infarct lesioning in the PLIC. Serial T2-weighted images were acquired $(\mathrm{TR}=5,620$ $\mathrm{ms}, \mathrm{TE}=113 \mathrm{~ms}, \mathrm{FOV}=96 \times 96 \mathrm{~mm}$, data matrix $256 \times 256 \mathrm{~mm}$, slice thickness $=1 \mathrm{~mm}$ ) and exported into a Dicom viewer (RadiAnt Dicom Viewer, Medixant, Poznan, Poland) for further analysis. The infarct area was visualized in three dimensions using multiplanar reconstruction (MPR).

\section{Statistical analysis}

All statistical analyses in this study were performed in Origin 8.5 software (OriginLab, Northampton, MA, USA). A two-way ANOVA was used for comparisons between groups or times. We performed a simple linear regression analysis to determine the statistical correlations between neurological assessment scores, brinkman board scores, and internal capsule infarct volume. Regression models were validated with an F-test $(\mathrm{p}<0.05)$.

\section{RESULTS}

\section{Neural tracing of hand motor fibers}

In this study, we used viral anterograde tracing to identify the course of hand motor fibers in the internal capsule. Neural tracing revealed that some of the hand motor fibers cross the midline through the corpus callosum with the remaining motor fiber entering the ipsilateral internal capsule (Fig. 1B). The hand motor fibers are densely distributed in the full length of the posterior 
A.

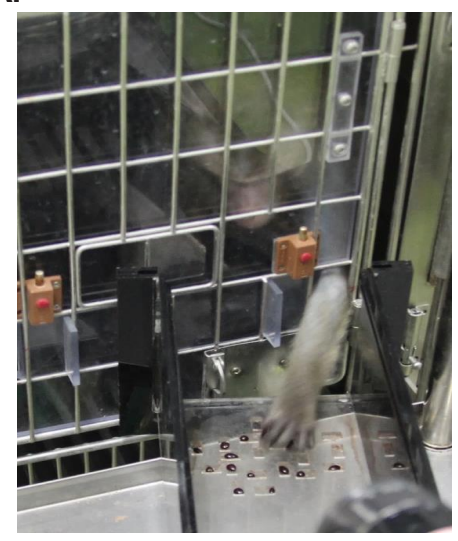

Brinkman board test
for left hand
B.

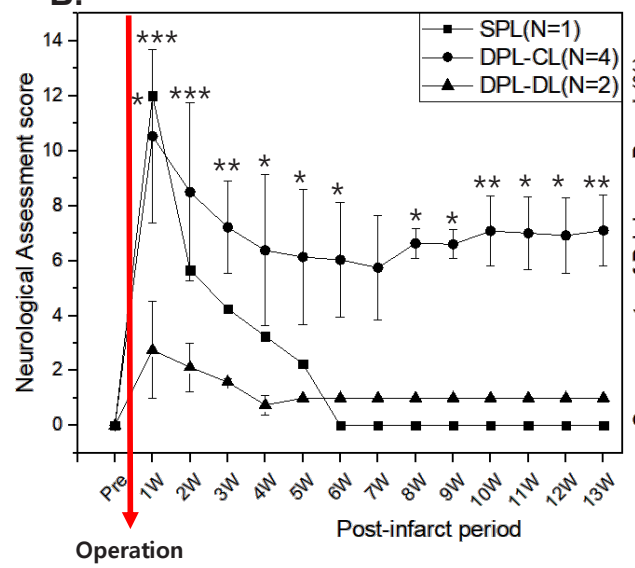

Longitudinal follow-up of neurological assessment scores
C.

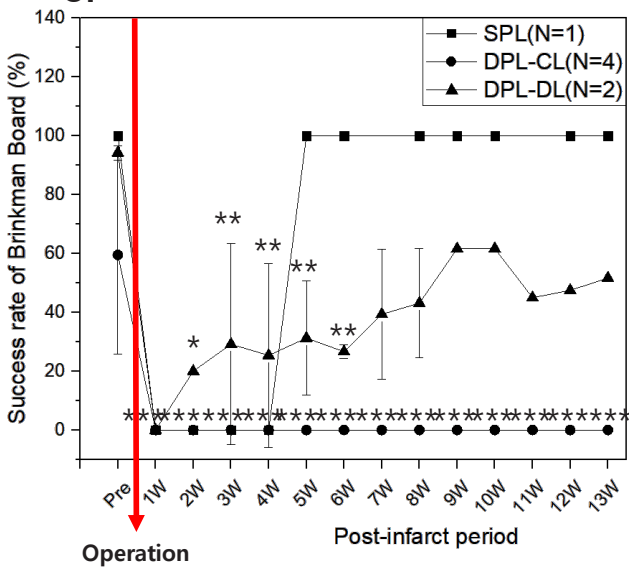

Brinkman board scores

Fig. 3. Behavioral testing and post-infarct behavioral changes. (A) The Brinkman board test. The macaqués left hand was allowed to extend through a small window to reach the pellets in the Brinkman board. (B) Longitudinal follow-up of neurological assessment scores following capsular infarct in each group. (C) Longitudinal follow-up of Brinkman board scores following capsular infarct in each group. ${ }^{* * *} \mathrm{p}<0.001,{ }^{* *} \mathrm{p}<0.01,{ }^{*} \mathrm{p}<0.05$.

portion of the PLIC (Fig. 1D). The distribution pattern of the corticospinal tract within the internal capsule is well known [20]. Hand motor fibers are thought to be located anterolateral to foot fibers and face motor fibers are located in the anteromedial portion of PLIC. However our neural tracing study shows that hand motor fibers are distributed in the full width of the PLIC, likely overlapping with leg motor fibers [21].

In addition, hand motor fibers follow the oblique shape of the internal capsule at the IC level, making it difficult to compromise the motor fibers with an orthogonal approach from above. Lateral tilting of the optical fiber by about 30 degrees appears to be the optimum angle to compromise the majority of hand motor fibers (Fig. 2B, 2C).

\section{Behavioral changes after photothrombotic capsular lesioning}

Neurological assessment scores (NAS) were significantly increased immediately after capsular lesioning in the SPL and DPLCL group (Fig. 3B), but not in the DPL-DL group.

Post-stroke NAS showed the increase of the scores exclusively in motor function such as movement of extremities, gait or grasp while the scores of consciousness, visual field, and neglect were not affected. Therefore, post-stroke NAS were below 14 in the SPL and DPL one week following stroke lesioning. Three weeks after infarct lesioning, animals in the SPL and DPL-DL groups showed improvement in NAS (to below 2) whereas the DPL-CL group showed significant maintenance of increased NAS above 6 .

All macaques showed an immediate significant decline in
Brinkman board scores (BBS) (Fig. 3C). The animal of SPL ( $\mathrm{n}=1$ ) showed the depressed mood following stroke lesioning and did not respond to the Brinkman board testing. However, it showed the sudden increase of BBS at 5 weeks. The DPL-DL groups showed a gradual improvement in BBS over the 3 months after the initial drop, whereas the impairment in the DPL-CL persisted over the entire 13-week observation period. This result shows that double-target infarct lesioning provides a persistent motor impairment, which can be used to reliably evaluate the effects of poststroke rehabilitation or neuromodulation.

\section{Extent of infarct lesioning}

Capsular infarct lesioning did not affect the general condition of the animals, such as body weight (Table 1). All macaques maintained their preoperative body condition. Histological examinations showed minor gliotic changes along the optical fiber tract in all macaques. Fig. 4 shows the extent of the infarct lesions in each group.

The infarct lesion in the SPL macaque was located correctly inside the internal capsule; however, the extent of the lesion was not sufficient to compromise the hand motor fibers in the PLIC (Fig. 4A). The two DPL-DL macaques had deviated lesioning that extended over both the PLIC and the internal globus pallidus (gray matter) away from the intended target; consequently, the lesions failed to destroy the full width of the arm motor fibers (Fig. 4B). Initially, we had technical difficulties in manufacturing the perfect holder of the optical fiber cannula, leading to these targeting de- 
A.



Single point lesion (SPL)
B.

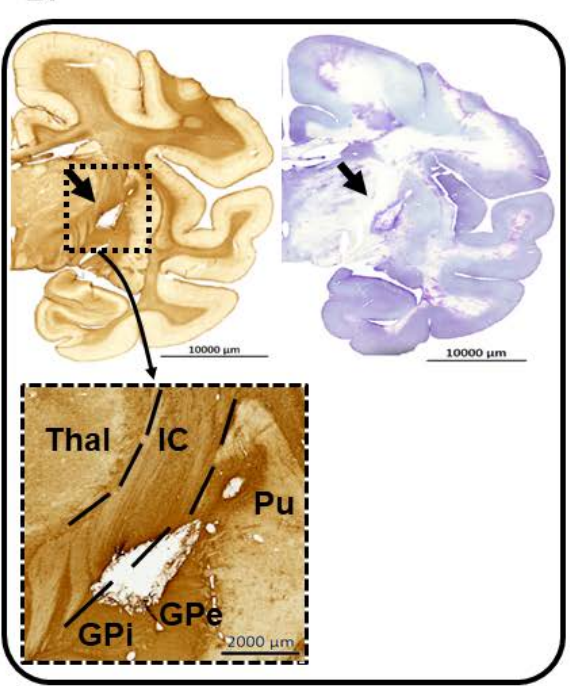

Double point lesion-deviated: (DPL)-DL
C.



Fig. 4. Histological sections showing the extent of the infarct lesion in each group. GFAP (left) and nissle (right) staining expressed an infarct lesion in the ipsi-lesional hemisphere $(\times 200$, top), and the enlarged images were shown at the bottom. Scale bars, 10,000 um (top), 2,000 um (bottom). (A) Single point lesion. (B) Double point lesion - deviated lesion group. (C) Double point lesion - correct lesion group. Thal, thalamus; IC, internal capsule, Pu, putamen; GPe, globus pallidus externus; GPi, globus pallidus internus.

viations. Four of the DPL macaques had infarct lesioning correctly located inside the PLIC (DPL-CL), compromising almost all of the hand motor fibers (Fig. 4C). Thus, this group had a significantly greater volume and extent of infarct lesioning than the other two groups.

Long-term follow-up MRI in two animals showed that the infarct lesions spanned the full length of the PLIC in the DPL animal but only part of the length of the PLIC in the SPL animal (Fig. 5).

MRI was helpful for identifying whether the lesion had been targeted correctly or not. However, in this study, the quality of MRI images of the infarct lesions was inferior to that from histological imaging (Fig. 5).

\section{Correlation between infarct volume and behavioral changes}

Fig. 6 shows the infarct volume in each group.

The volumes in the SPL, DPL-DL, and DPL-CL groups were $4.747 \mathrm{~mm}^{3}, 6.227 \pm 4.35 \mathrm{~mm}^{3}$, and $21.435 \pm 17.4 \mathrm{~mm}^{3}$, respectively. There was a significant difference in infarct volume between the DPL-CL and DPL-DL groups. This finding is compatible with the results from our previous study indicating that photothrombotic infarct lesioning produces a larger area of infarct (internal capsule) in the white matter (internal capsule) than in the neighboring gray matter. Although the environment and the doses of rose bengal dye and light irradiation were the same in the two groups, a larger infarct area was produced in the DPL-CL group (where the lesion was restricted to the white matter) than in the DPL-DL group (where the lesion extended over both white and gray matter).

Fig. 7 shows the correlation between infarct volume and postinfarct behavioral changes.

As the infarct volume increased, the neurological assessment scores increased $\left(R^{2}=0.5731, \mathrm{p}<0.05\right)$ and the Brinkman board scores decreased $\left(R^{2}=0.5958, p<0.05\right)$. This suggests that infarct volume was the critical factor in determining post-infarct behavioral changes (Fig. 7). Additional strategies to increase the infarct volume within the PLIC should thus be considered.

\section{DISCUSSION}

In this study, we demonstrated that a small photothrombotic lesion in the internal capsule can generate a persistent motor deficit in a gyrencephalic macaque, the cynomolgus monkey, if the lesion encompasses the full length of the PLIC. Although the internal capsule infarct volume produced with this approach was much lower than with other types of lesioning [22-24], accurate photothrombotic lesioning with stereotactic placement of the optical fiber parallel to the oblique course of the internal capsule was able to achieve long-term motor deficits. In addition, the macaques tolerated the operative procedures with zero mortality and reduced morbidity.

A reproducible infarct model with long-term motor deficits is essential for the development of rehabilitative interventions 


\section{A. DPL $(\mathrm{N}=1)$}

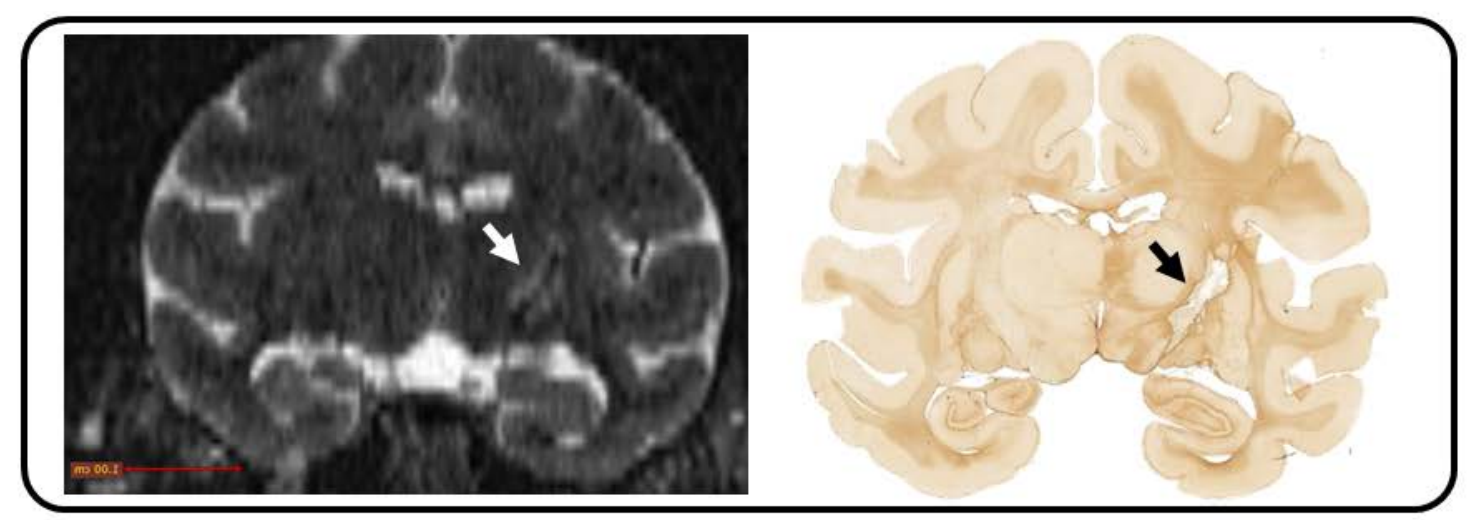

\section{B. $S P L(N=1)$}

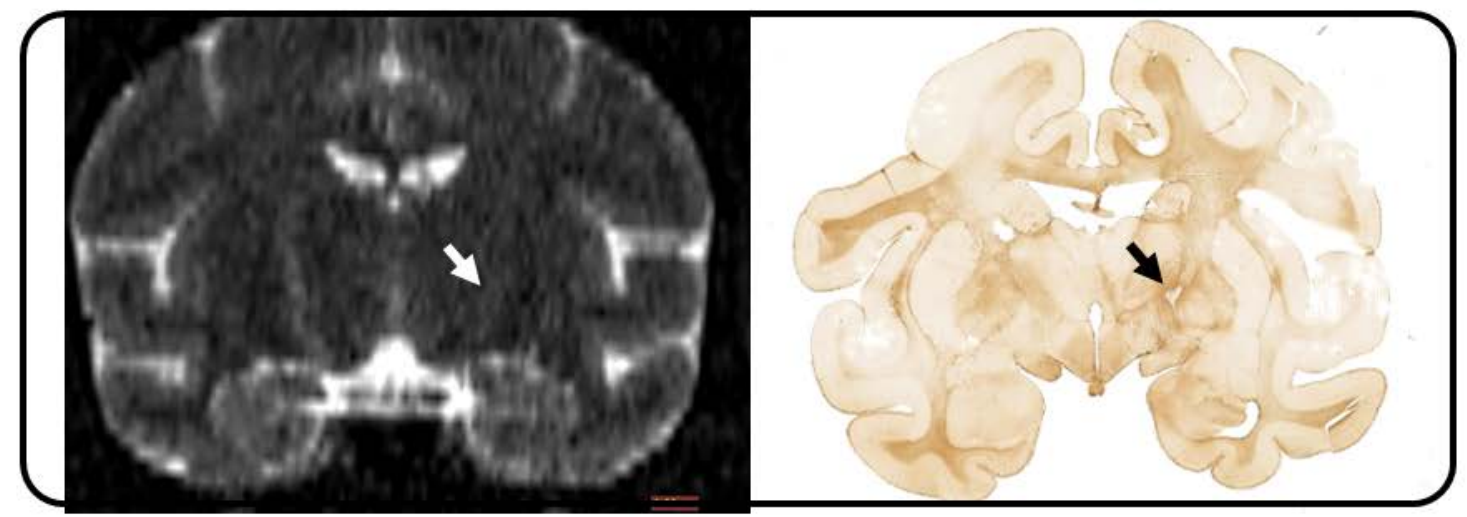

Fig. 5. Long-term follow-up MRI and histological imaging for double- versus single-point capsular infarct lesions. (A) Double-point lesion visualized with MRI (left) and glial fibrillary acidic protein staining (right). (B) Single-point lesion visualized with MRI (left) and glial fibrillary acidic protein staining (right).

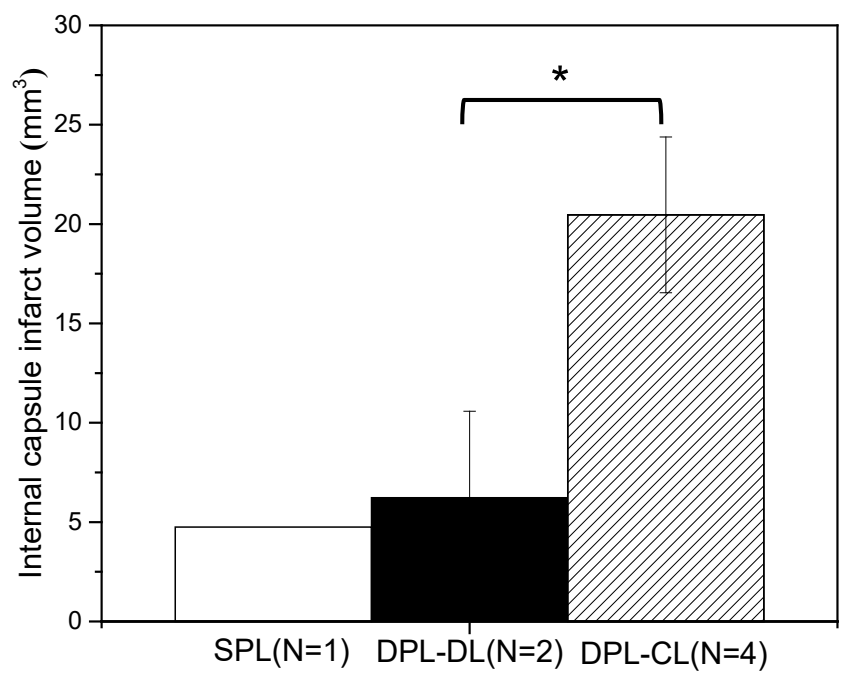

Fig. 6. Infarct volume in each group. $\mathrm{p}<0.05$.

for chronic stroke. When an NHP model is used, high levels of reproducibility and survival are required to minimize the sacrifice of valuable animals [23]. In our previous reports, we showed that a circumscribed infarct lesion via stereotactically guided photothrombotic lesioning of the internal capsule can generate a reproducible white-matter stroke model with persistent motor deficits in rats $[9,10,25]$. Here, we applied similar techniques to the macaque brain to produce to produce a persistent hand motor deficit. Our results demonstrate that the photothrombotic infarct procedure can be applied successfully to gyrencephalic brains to destroy motor fibers. However, the partial lesioning produced by a single-point lesion was not sufficient to produce a persistent motor deficit in macaques. Double-point lesioning that encompassed the entire length of the PLIC was necessary to achieve a long-term motor deficit. Furthermore, the PLIC is very narrow so lesions are likely to miss the target even when stereotactic techniques are used. To address this, we used an "incline adaptor" with our stereotactic apparatus so that the cannula was introduced parallel to the course of the hand motor fibers [17]. The combination of double-point lesioning with an incline adaptor worked well to produce a PLIC infarct of sufficient size and precision to induce a persistent deficit.

We performed neural tracing to accurately localize the course of hand motor fibers inside the PLIC. The results showed that hand motor fibers are distributed widely across the whole length of the PLIC. Further, the double-point lesions produced motor deficits in 

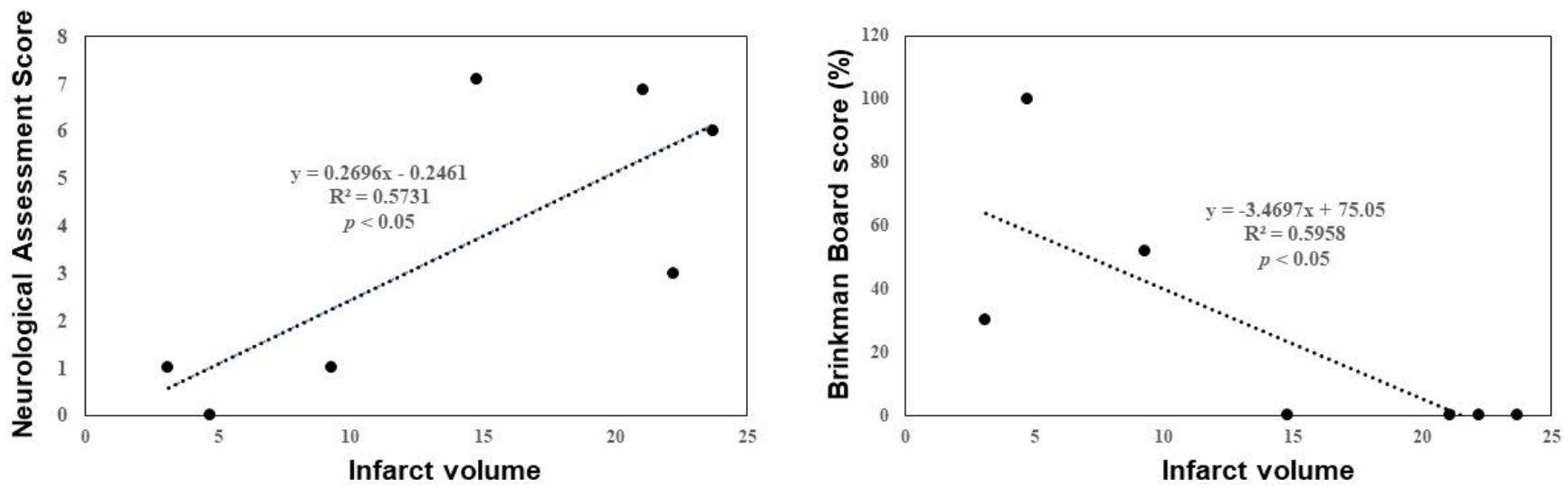

Fig. 7. Correlation between infarct volume and behavioral scores. (A) $\mathrm{R}^{2}$ value of 0.5731 between PLIC infarct volume and neurological assessment score $(\mathrm{p}<0.05)$. (B) $\mathrm{R}^{2}$ value of 0.5958 between PLIC infarct volume and success rate with the Brinkman board $(\mathrm{p}<0.05)$.

the foot as well as the hand, leading to walking deficits. These findings suggest that the hand motor fibers overlap with the leg motor fibers in the PLIC [21]; therefore, selective lesioning for hand or leg motor fibers was not possible in our study. Nonetheless, it was feasible to produce a persistent, contralateral motor deficit following destruction of the PLIC.

The PLIC infarct volume was very small in this study compared with the volumes produced by other methods of capsular infarct modeling. The average infarct volume in the macaques with DPLDL was $21.435 \pm 17.4 \mathrm{~mm}^{3}$, with no perioperative mortality or morbidity. Traditional ligation of the lenticulostriate artery or the anterior choroidal artery produces an infarct in the internal capsule; however, the damage extends to other areas including the basal ganglia, the optic tract, and the centrum semiovale [23, 24]. Injections of the vasoconstrictor endothelin-1 (ET-1) can be targeted to the desired site: however, ET-1 is likely to diffuse out to neighboring tissue, leading to greater-than-expected infarct volumes. These larger infarct volumes are likely to result in higher levels of perioperative mortality and morbidity. Furthermore, a larger infarct volume does not guarantee the greater severity in motor deficit that is essential for rehabilitation intervention studies. Small but accurately targeted PLIC infarcts are the better option for producing a capsular infarct model with a persistent motor deficit and lower mortality and morbidity.

This study has several limitations. First, we had a limited number of macaques in each group: considering the value of gyrencephalic macaques, we had to limit the number of macaques in a group if the infarct lesioning produced a smaller effect than expected (e.g., the SPL animal). We used the limited results to adjust our experimental procedures (e.g., switching to double-point lesioning). Second, photothrombotic infarct lesioning does not mimic the occlusion of end arteries that is typical of lacunar infarct. However, occlusion of lenticulostriate arteries or anterior choroidal artery produced the variable extent of infarct and neurological impairment due to its anatomical variabilities [23, 24]. Further, the selective destruction of white matter in this study can be used to assess the unique pathophysiology and recovery mechanisms of whitematter stroke. These types of research, coupled with gray-matter stroke research, will shed light on the entire spectrum of stroke.

In conclusion, the precise photothrombotic infarction technique can be applied to gyrencephalic macaques to produce a model of capsular infarct. This model may help overcome the translational barriers that currently limit investigations into the mechanisms of post-stroke recovery and the changes in brain activity and behavior that occur with white-matter stroke.

\section{ACKNOWLEDGEMENTS}

This work was supported by the grants from Korea Institute of Toxicology (KIT, Korea) and Basic science Research Program funded by the Ministry of science and ICT [Project number: KK1613-02, KK-1717-01, NRF-2019M3C1B8090841] and 2021 Joint Project of GIST.

\section{REFERENCES}

1. Norrving B (2003) Long-term prognosis after lacunar infarction. Lancet Neurol 2:238-245.

2. Valdés Hernández MDC, Grimsley-Moore T, Chappell FM, Thrippleton MJ, Armitage PA, Sakka E, Makin S, Wardlaw JM (2021) Post-stroke cognition at 1 and 3 years is influenced by the location of white matter hyperintensities in patients with lacunar stroke. Front Neurol 12:634460.

3. Benavente OR, Pearce LA, Bazan C, Roldan AM, Catanese L, 
Bhat Livezey VM, Vidal-Pergola G, McClure LA, Hart RG; SPS3 Investigators (2014) Clinical-MRI correlations in a multiethnic cohort with recent lacunar stroke: the SPS3 trial. Int J Stroke 9:1057-1064.

4. Schiemanck SK, Kwakkel G, Post MW, Kappelle LJ, Prevo AJ (2008) Impact of internal capsule lesions on outcome of motor hand function at one year post-stroke. J Rehabil Med 40:96-101.

5. Sozmen EG, Kolekar A, Havton LA, Carmichael ST (2009) A white matter stroke model in the mouse: axonal damage, progenitor responses and MRI correlates. J Neurosci Methods 180:261-272.

6. Kaur H, Prakash A, Medhi B (2013) Drug therapy in stroke: from preclinical to clinical studies. Pharmacology 92:324334.

7. Sozmen EG, Hinman JD, Carmichael ST (2012) Models that matter: white matter stroke models. Neurotherapeutics 9:349358.

8. Song YM (2007) Somatotopic organization of motor fibers in the corona radiata in monoparetic patients with small subcortical infarct. Stroke 38:2353-2355.

9. Kim HS, Kim D, Kim RG, Kim JM, Chung E, Neto PR, Lee MC, Kim HI (2014) A rat model of photothrombotic capsular infarct with a marked motor deficit: a behavioral, histologic, and microPET study. J Cereb Blood Flow Metab 34:683-689.

10. Kim D, Kim RG, Kim HS, Kim JM, Jun SC, Lee B, Jo HJ, Neto PR, Lee MC, Kim HI (2015) Longitudinal changes in restingstate brain activity in a capsular infarct model. J Cereb Blood Flow Metab 35:11-19.

11. Kim RG, Cho J, Ree J, Kim HS, Rosa-Neto P, Kim JM, Lee MC, Kim HI (2016) Sensory-parietal cortical stimulation improves motor recovery in severe capsular infarct. J Cereb Blood Flow Metab 36:2211-2222.

12. Cho J, Kwon DH, Kim RG, Song H, Rosa-Neto P, Lee MC, Kim HI (2016) Remodeling of neuronal circuits after reach training in chronic capsular stroke. Neurorehabil Neural Repair 30:941-950.

13. Nam MH, Cho J, Kwon DH, Park JY, Woo J, Lee JM, Lee S, Ko HY, Won W, Kim RG, Song H, Oh SJ, Choi JW, Park KD, Park EK, Jung H, Kim HS, Lee MC, Yun M, Lee CJ, Kim HI (2020) Excessive astrocytic GABA causes cortical hypometabolism and impedes functional recovery after subcortical stroke. Cell Rep 32:107861.
14. Lee MC, Kim RG, Lee T, Kim JH, Lee KH, Choi YD, Kim HS, Cho J, Park JY, Kim HI (2020) Ultrastructural dendritic changes underlying diaschisis after capsular infarct. J Neuropathol Exp Neurol 79:508-517.

15. Smith Y, Wichmann T, DeLong MR (2014) Corticostriatal and mesocortical dopamine systems: do species differences matter? Nat Rev Neurosci 15:63.

16. Cook DJ, Tymianski M (2012) Nonhuman primate models of stroke for translational neuroprotection research. Neurotherapeutics 9:371-379.

17. Kim HS, Byun D, Kim RG, Kang GH, Park JY, Yang YS, Han SC, Kim HI (2018) Simplified adaptor for stereotactic surgery in non-human primates. J Neurosci Methods 295:139-143.

18. Roitberg B, Khan N, Tuccar E, Kompoliti K, Chu Y, Alperin N, Kordower JH, Emborg ME (2003) Chronic ischemic stroke model in cynomolgus monkeys: behavioral, neuroimaging and anatomical study. Neurol Res 25:68-78.

19. Schmidlin E, Kaeser M, Gindrat AD, Savidan J, Chatagny P, Badoud S, Hamadjida A, Beaud ML, Wannier T, Belhaj-Saif A, Rouiller EM (2011) Behavioral assessment of manual dexterity in non-human primates. J Vis Exp 57:3258.

20. Holodny AI, Gor DM, Watts R, Gutin PH, Ulug AM (2005) Diffusion-tensor MR tractography of somatotopic organization of corticospinal tracts in the internal capsule: initial anatomic results in contradistinction to prior reports. Radiology 234:649-653.

21. Duerden EG, Finnis KW, Peters TM, Sadikot AF (2011) Three-dimensional somatotopic organization and probabilistic mapping of motor responses from the human internal capsule. J Neurosurg 114:1706-1714.

22. Murata Y, Higo N (2016) Development and characterization of a macaque model of focal internal capsular infarcts. PLoS One 11:e0154752.

23. Yonas H, Wolfson SK Jr, Dujovny M, Boehnke M, Cook E (1981) Selective lenticulostriate occlusion in the primate. A highly focal cerebral ischemia model. Stroke 12:567-572.

24. Puentes S, Kaido T, Hanakawa T, Ichinohe N, Otsuki T, Seki $\mathrm{K}$ (2015) Internal capsule stroke in the common marmoset. Neuroscience 284:400-411.

25. Song H, Jung W, Lee E, Park JY, Kim MS, Lee MC, Kim HI (2017) Capsular stroke modeling based on somatotopic mapping of motor fibers. J Cereb Blood Flow Metab 37:29282937. 